\title{
Fibroscan Compared to FIB-4, APRI, and AST/ALT Ratio for Assessment of Liver Fibrosis in Saudi Patients With Nonalcoholic Fatty Liver Disease
}

\author{
Hind I. Fallatah, ${ }^{1,}$ Hisham O. Akbar, ${ }^{1}$ and Alyaa M. Fallatah ${ }^{2}$ \\ ${ }^{1}$ Department of Internal Medicine, Unit of Hepatology, Saudi Arabia Hospital Jeddah, King Abdulaziz University, Jeddah, Saudi Arabia \\ ${ }^{2}$ Department of Medicine, King Fahd Military Hospital, Jeddah, Saudi Arabia \\ "Corresponding author: Hind I. Fallatah, Department of Internal Medicine, King Abdulaziz University, Jeddah, Saudi Arabia. E-mail: hindfallatah@hotmail.com
}

Received 2016 April 09; Revised 2016 May 22; Accepted 2016 June 14.

\begin{abstract}
Background: Nonalcoholic fatty liver disease (NAFLD) is being increasingly recognized as a cause of chronic liver disease. It has also been associated with devastating outcomes such as decompensated liver cirrhosis and hepatocellular carcinoma, as well as diabetes and metabolic syndrome.

Objectives: This study was conducted in order to assess liver fibrosis using Fibroscan, and to compare these results to the use of Fibrosis-4 (FIB-4) scores, AST platelet ratio index (APRI scores), and the AST/ALT ratios on NAFLD patients.

Patients and Methods: A cross sectional study study was conducted on NAFLD patients who underwent Fibroscan examinations between September 1, 2011 and June 30, 2014. Demographic data was collected, including sex, age, and nationality; serum alanine aminotransferase levels (ALT, 30 - $65 \mathrm{U} / \mathrm{L}$ ), serum aspartate aminotransferase levels (AST, 15 - $37 \mathrm{U} / \mathrm{L}$ ), and platelet counts (150 - 400 $\mathrm{k} / \mu \mathrm{L}$ ) were also determined. The stages of fibrosis (F0 1 - 6, F1 6.1 - 7, F2 7 - 9, F3 9.1 - 10.3, and F4 $\geq 10.4$ ) were defined in kPa. For each patient, the AST/ALT ratio was also measured. The results of APRI and FIB-4 were compared with the Fibroscan fibrosis scores.

Results: The results of 122 patients were analyzed, including 65 (53.3\%) males with a mean age of 50.2 years (SD: 13.7; range: 18 - 86). The males were significantly younger than the females (48.7 years (SD: 16.03) versus 51.8 years (SD: 10.3 P $=0.05$ ), respectively). The mean stiffness score was 12.02 (SD: 12.7) kPa. Forty-four patients (36\%) had advanced fibrosis. The mean platelet and serum ALT levels were normal. There was a significant positive correlation between the Fibroscan results and the AST/ALT ratios, the APRI scores, and the FIB- 4 results. Similarly, there was a significant positive correlation between age and fibrosis score, and a significant negative correlation between platelet count and stiffness score.

Conclusions: The data showed that more than one-third of the cohort exhibited advanced fibrosis, demonstrating the need for the early diagnosis and treatment of NAFLD. The use of Fibroscan with other serum markers has been shown to be helpful for the diagnosis of severe fibrosis.
\end{abstract}

Keywords: NAFLD, Saudi Arabia, Diabetes, Liver Fibrosis, Stiffness Measurements

\section{Background}

Nonalcoholic fatty liver disease (NAFLD) is increasingly recognized as a cause of chronic liver disease, and often results in the devastating outcomes of decompensated liver cirrhosis and hepatocellular carcinoma (1-4). Compared with the risks of the general population, NAFLD is also associated with increased risks of mortality due to liver disease and cardiovascular disease (2-7). The reported prevalence of this disease varies among studies, from $12.5 \%$ to $51 \%$, which may reflect the variability in NAFLD risk factors among the studied populations along with the particular diagnostic methods used $(1,2,8)$. NAFLD is commonly associated with diabetes and is an important feature of metabolic syndromes and insulin resistance $(1,4$, $8-10)$. The spectrum of NAFLD severity is variable, ranging from mild symptoms to differing degrees of inflammation, nonalcoholic steatohepatitis (NASH), or a severe form of decompensated liver cirrhosis which occurs in a minority of patients (1-4). It is frequently diagnosed in patients with liver disease in Saudi Arabia $(11,12)$.

The assessment of liver fibrosis is essential for predicting the prognosis and outcome of all forms of chronic liver disease. A liver biopsy is the gold standard for the assessment of liver fibrosis, but it has its limitations, which include life-threatening complications $(13,14)$. Alternative methods of non-invasive laboratory and radiological testing for the assessment of liver fibrosis in NAFLD have evolved during the past decade, and these methods may be able to overcome the limitations of liver biopsy $(13,14)$. These methods include the AST/ALT ratio, the AST platelet ratio index (APRI), and the Fibrosis 4 (FIB-4) score (13-18). 
Transient elastography (Fibroscan), an ultrasound-based technique, is one of the most extensively used and wellvalidated non-invasive methods for the assessment of liver fibrosis (14, 19-22). However, the diagnostic yield of Fibroscan is limited by obesity in approximately one-third of patients. This limitation has recently been improved by the design of an XL probe for patients with body mass indices (BMIs) of greater than $30 \mathrm{~kg} / \mathrm{m}^{2}$, which has an accuracy rate similar to that of the M probe $(14,23,24)$.

\section{Objectives}

In this study, the results of liver fibrosis assessments using Fibroscan are compared to the FIB-4 scores, APRI scores, and AST/ALT ratios for NAFLD patients who were diagnosed and followed-up on at the hepatology clinic of King Abdulaziz University hospital (KAUH).

\section{Patients and Methods}

All ethical guidelines for research were followed, and the approval of the KAU ethical committee was obtained for this study.

\subsection{Study Period, Population, and Design}

This was a cross-sectional study conducted from September 1, 2011 through June 30, 2014. The study population included patients who were diagnosed with NAFLD or nonalcoholic steatohepatitis (NASH) based on abdominal ultrasound examination and serum liver enzyme testing performed at the KAUH hepatology clinic. Patients who were included had available ultrasound and blood test results in the hospital information system and had undergone Fibroscan examinations within the study period. Those patients with incomplete data were excluded from analysis. Patients were also excluded if they had evidence of other chronic liver diseases, including hepatitis B or C, or autoimmune hepatitis (AIH) (as indicated by testing positive for autoimmune antibodies or demonstrating a favorable response to steroids), or alcoholic liver disease. Patients on hepatotoxic medications, such as chronic methotrexate, were also excluded, as well as those with advanced liver disease, cardiac failure, and hepatic congestion. In addition, those who could not undergo Fibroscan examinations because of very high BMIs or for other reasons, and those with clinical or ultrasound evidence of decompensated cirrhosis, were also prevented from participating in the study.

Demographic data on sex, age, and nationality were obtained for each patient. The laboratory test results that were evaluated included those in the hospital information system. All results were obtained within one month of a Fibroscan examination.

The laboratory reference normal range of serum alanine aminotransferase (ALT) at KAUH is $30-65 \mathrm{U} / \mathrm{L}$, and it is measured using a method reported in a previous study performed at our center (25). Normal upper serum ALT limits were defined as $45.25 \mathrm{U} / \mathrm{L}$ for males and $30.47 \mathrm{U} / \mathrm{L}$ for females (25). The serum aspartate aminotransferase (AST) normal reference range is $15-37 \mathrm{U} / \mathrm{L}$, and the normal reference range for platelet counts is $150-400 \mathrm{k} / \mu \mathrm{L}$.

Liver enzymes were measured using a dimension clinical chemistry system (Flex Reagent Cartridge). For each patient, the AST/ALT ratio was measured, and the APRI score was determined by using the following equation (Equation 1) (19):

APRI $=\frac{\text { AST Level } /(\text { ULN })}{\text { Platelet } C \text { ounts }\left(10^{9} / L\right) \times 100}$

FIB-4 was determined by using the following formula (Equation 2)(21):

$\mathrm{FIB}-4=\frac{\operatorname{Age}(\mathrm{y}) \times \operatorname{AST}(\mathrm{U} / \mathrm{L})}{\text { Platelet Count } 10^{9} / L \times \sqrt{\operatorname{ALT}(\mathrm{U} / \mathrm{L})}}$

A Fibroscan 502 (2005), manufactured by Echosens (42 Rue Monge 74005, Paris, France), was used in the study. The manufacturer's guidelines and findings from previous studies have indicated that 10 valid Fibroscan readings are necessary for a test to be deemed successful (20-22). Greater than $70 \%$ success rates were achieved for all of the patients included in the study. Each Fibroscan examination was performed by the two investigators, Dr. Hind and Professor Hisham. The following stages of fibrosis were defined based on several previous studies and the references provided by the manufacturer: F0 1 - 6, F1 6.1 - 7, F2 7 - 9, F3 $9.1-10.3$ and $\mathrm{F} 4 \geq 10.4(1-4)$.

\subsection{Statistical Analysis}

The IBM statistical package for the social sciences (SPSS) version 20 was used for the analysis. Descriptive data was generated and a Student's t-test was used to compare the differences in the mean ages and serum ALT values between the males and the females. Correlation analysis was used to compare the degree of hepatic fibrosis with age, platelet count, and serum ALT level. A simple dots graph was used to determine the correlation between the Fibroscan results and the AST/SLT ratios, the APRI scores, and the FIB-4 scores. A Student's t-test was used to compare the AST/ALT ratio to the APRI and FIB-4 scores between patients with advanced fibrosis higher than F2 and patients with mild to moderate fibrosis of F2 or less. 


\section{Results}

Each year, approximately 400 successful Fibroscan examinations are performed at KAUH, approximately $15 \%$ of which are performed on NAFLD patients. After excluding patients according to the above-mentioned criteria, 122 patients were included in the final analysis, including 65 (53.3\%) males and 57 (46.7\%) females. The mean age was 50.2 years (SD: 13.7, range: 18 - 86). The male patients were significantly younger than the female patients (48.7 years (SD: 16.03) versus 51.8 years (SD:10.3), respectively, $\mathrm{P}=0.002$ )

The mean stiffness score was 12.02 (SD: 12.7) kPa, and most patients exhibited either no fibrosis or an advanced level of the disease (Table 1 shows the distribution of patients according to the stage of fibrosis). Male patients exhibited higher stiffness scores than female patients, but this difference was not significant (13.6(SD:14.5) versus 10.2 (SD:10.02), respectively, $\mathrm{P}=0.061$ ). In addition, the male patients exhibited significantly higher mean serum ALT levels compared with the female patients (73.5 (SD: 58) versus 44.7 (SD:36), respectively, $\mathrm{P}=0.039$ ) (see Table 2 for ALT and platelet counts).

Table 1. Distribution of Patients According to the Stage of Liver Fibrosis

\begin{tabular}{|lc}
\hline Stage of Fibrosis & No. $(\%)$ \\
\hline Fo $(\mathbf{0}-5.9)$ & $52(42.6)$ \\
\hline F1 $(6-6.9)$ & $15(12.3)$ \\
\hline F2 $(7-9)$ & $11(9.0)$ \\
\hline F3 $(9.1-10.3)$ & $4(3.3)$ \\
\hline F4 $(\geq 10.4)$ & $40(32.8)$ \\
\hline Total & $122(100.0)$ \\
\hline
\end{tabular}

Table 2. Mean Platelet Counts, Serum Alanine Transaminase Levels, and Aspartate Aminotransferase Levels

\begin{tabular}{lcc}
\hline Lab Test & Mean \pm SD & Normal Reference Range \\
\hline Platelets, $\mathbf{k} / \mu \mathbf{L}$ & $239.85 \pm 88.8$ & $15-400$ \\
\hline Serum ALT, U/L & $58.4 \pm 49.8$ & $30-65$ \\
\hline Serum AST, U/L & $42.2 \pm 47.6$ & $15-37$ \\
\hline
\end{tabular}

Correlation analysis showed a significant positive correlation between age and fibrosis scores $(r=0.27,0.220$, and 0.313 with $\mathrm{P}=0.004,0.001$, and 0.001 for Pearson, Kendall, and Spearman correlations, respectively). On the other hand, a significant negative correlation between platelet count and stiffness scores was obtained $(\mathrm{r}=$ $0.315,-0.283$, and -0.41 with $\mathrm{P}=0.001,<0.001$, and $<0.001$ for Pearson, Kendall, and Spearman correlations, respectively). Serum ALT level was determined to be significantly negatively correlated with age by using both the Kendall and Spearman correlations $(\mathrm{r}=-0.17$ and -0.232 , and $\mathrm{P}=$ 0.0155 and 0.022 , respectively). A significant positive correlation was observed between serum ALT and hepatic stiffness measurements using both the Kendall and Spearman correlations $(\mathrm{r}=0.202$ and 0.284 , and $\mathrm{P}=0.004$ and 0.005 , respectively). There was a significant difference in the results of the stiffness scores for APRI and the FIB-4 calculations between patients with advanced fibrosis of more than F2 at 44 (36\%) and those with mild to moderate fibrosis of F2 or under at 78 (64\%) (Table 3 ).

The fibrosis scores on the Fibroscan were significantly correlated with the AST/ALT, APRI, and FIB-4 values (Figures $1-3$, respectively).

Table 3. Differences in Stiffness Scores, Aspartate Aminotransferase/Alanine Transaminase Ratio, Aspartate Aminotransferase to Platelet Ratio Index, and FIB-4 Scores Between Patients With Mild to Moderate Fibrosis and Those With Advanced Fibrosis $^{\mathrm{a}}$

\begin{tabular}{lccc}
\hline & $>$ F2 $(\mathbf{N}=\mathbf{4 4})$ & F2 or Less $(\mathbf{N}=\mathbf{7 8})$ & PValue \\
\hline Stiffness score & $23.7 \pm 15$ & $5.33 \pm 1.6$ & $<0.001$ \\
AST/ALT & $0.87 \pm 0.36$ & $0.72 \pm 0.57$ & 0.67 \\
APRI & $1.1 \pm 1.04$ & $0.314 \pm 0.3$ & $<0.001$ \\
FIB-4 & $2.64545 \pm 2.1$ & $0.93048 \pm 0.823436$ & $<0.001$ \\
\hline
\end{tabular}

${ }^{\mathrm{a}}$ Values are expressed as mean $\pm \mathrm{SD}$.

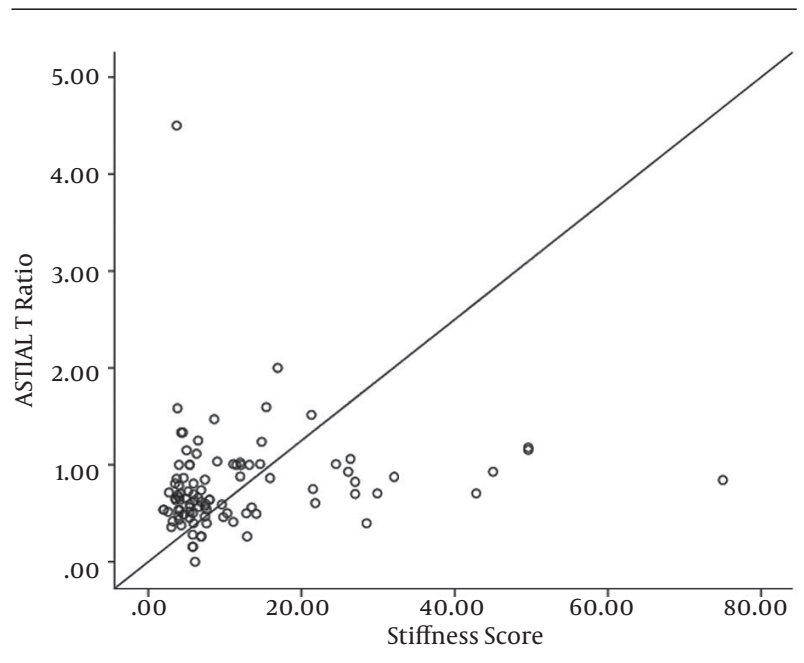

Figure 1. Correlation Between Fibrosis Scores on Fibroscan and AST/ALT Ratios ( $r=$ $0.23, \mathrm{P}=0.022$ ) 


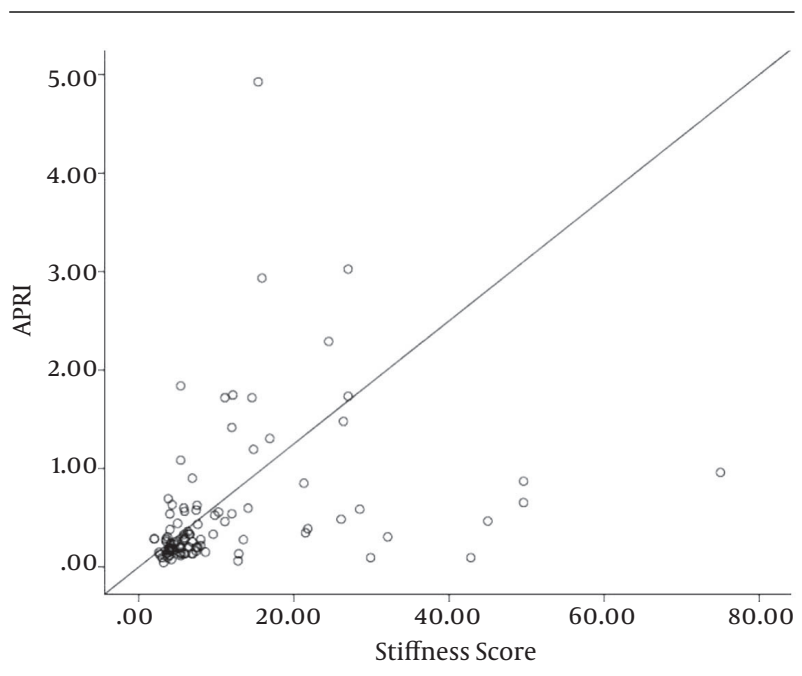

Figure 2. Correlation Between Fibrosis Scores on Fibroscan and APRI Scores $(r=0.51$, $\mathrm{P}>0.001)$

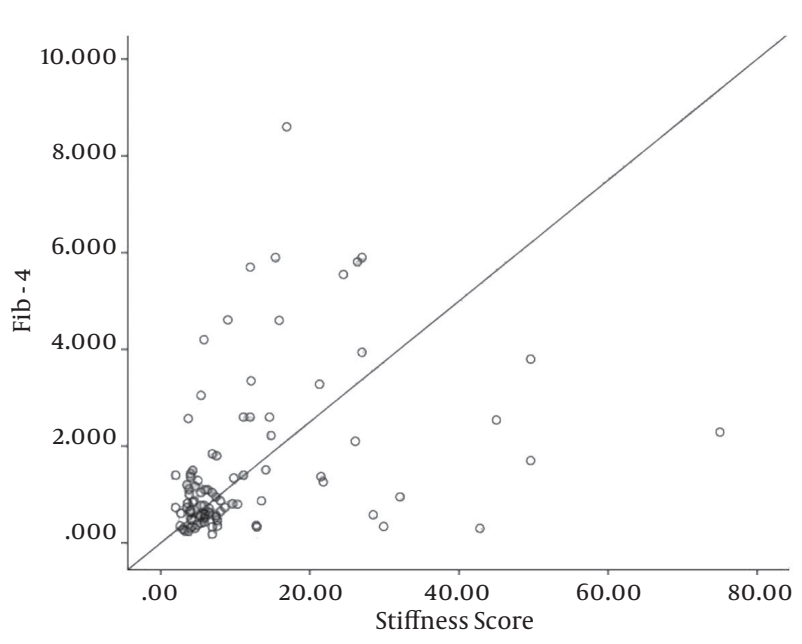

Figure 3. Correlation Between Fibrosis Scores on Fibroscan and FIB-4 Scores $(r=0.5$, $\mathrm{P}>0.001)$

\section{Discussion}

The data showed that a high percentage of the NAFLD patients exhibited advanced stages of liver fibrosis based on the Fibroscan examinations. These results were supported by the strong correlation between the Fibroscan results and the AST/ALT ratio, APRI scores, and FIB-4 scores. Moreover, there was a strong negative correlation between platelet count and stiffness, as thrombocytopenia in liver disease is associated with advanced fibrosis $(26,27)$. The rate of advanced NAFLD determined in this study is higher than those reported by other authors using Fibroscan examinations to assess liver fibrosis $(20,22,24,26,28-31)$, and this rate is particularly alarming for the Saudi population, which has a high prevalence of type II diabetes and metabolic syndrome (11, 30-33).

All patients in the cohort for this study had baseline abdominal ultrasound examinations that showed steatosis; however, the ultrasound alone is not efficient in the assessment of advancement of liver disease. Razavizade et al. showed that the addition of serum markers to ultrasound examinations can help in categorizing NAFLD patients into mild against moderate or severe categories of NAFLD (34). This determination could also support the use of simple biomarkers in addition to ultrasound of the abdomen in the assessment of NAFLD when more advanced methods such as transient elastography or MRI elastography are not available, or when liver biopsy is not indicated.

It was also shown that male patients were more likely to suffer from advanced fibrosis at a younger age than female patients, which is not surprising given that male patients generally tend to have more severe liver diseases of most etiologies compared with females $(35,36)$. This phenomenon may be explained by the protective effect of female sex hormones on the progression of hepatic fibrosis (26). The center at KAUH previously published research findings as well as national and international data on NAFLD that have shown that males are more commonly affected than females $(1,2,5,8,11,12,21)$. Furthermore, the data revealed that age was associated with more advanced stages of the disease, which is consistent with previous NAFLD studies (1, 6-8).

Several studies on the general population have shown that serum ALT levels increase with advanced age $(25,37)$. However, our results showed an inverse relationship between ALT levels and age. Similar findings have been reported by Al- Hamoudi et al. in their analysis of NAFLD in Saudi Arabia (12). This altered relationship between serum ALT levels and age might be due to disease activity and the development of NASH in individuals of younger ages, which may lead to cirrhosis and the normalization of ALT at later ages $(1,3,9,10)$. A large number of NAFLD patients exhibited normal or near-normal liver enzyme levels, which has also been previously demonstrated in the Saudi population $(4,5,8,11,12,38)$.

The AST/ALT ratio was the least likely among the four non-invasive methods in this study to indicate a difference between mild to moderate and advanced fibrosis. Therefore, Fibroscan examinations, APRI scores, and FIB-4 scores can be used in the follow-up on early-stage NAFLD patients when liver biopsy has no clear indication. In addition, these non-invasive testing methods can be used for followup on patients who have had bariatric surgery or other treatment for NAFLD.

The pathogenesis of NAFLD has been explained by sev- 
eral theories, and the commonly accepted one is the "two hit theory" (39). The first "hit" is considered to be hepatic steatosis, and the second "hit" is inflammation and liver injury. Both hits are promoted by adipocytokines $(39,40)$. Recent data by Razavizade et al. has shown that serum adipokines such as visfatin, IL-6, TNF-a, TGF- $\beta 1$, and IL-8 were associated with a high probability of developing NAFLD and NASH $(41,42)$. Hence, these adipokines can be used as future biomarkers for assessment of NAFLD patients. Similar to previous studies $(24,25)$, the XL probe used at the study center since early 2012 has allowed for the successful examination of patients for whom accurate readings could not be obtained with the M probe.

\subsection{Conclusions}

This study has shown that the combination of Fibroscan and AST/ALT, APRI, and FIB-4 methods provides a valuable approach for assessing liver fibrosis in NAFLD patients. This can eliminate the need for liver biopsy in patients without clear indication. In addition, several recent studies have also validated the used of non-invasive markers in the diagnosis of NAFLD. The high proportion of the patients in this study with advanced fibrosis is alarming because the Saudi population exhibits a high prevalence of diabetes and metabolic syndrome. The establishment of a national program for the recognition of NAFLD is therefore essential to reduce the risk of liver disease progression.

The limitations of the study are as follows: 1) The retrospective nature of the study might adversely affect the reliability of the results, and a similar well-planned prospective study might be better to eliminate the selection bias; 2) The number of included patients might be small in view of the national NADF prevalence data, but this could be compensated for by the strict inclusion criteria; 3) Liver biopsy, the gold standard of NAFLD diagnosis, was not used in this study, but due to the complications such a procedure can cause, it should not be recommended for every patient with NAFLD.

\section{Acknowledgments}

We appreciate and are thankful for the supportive work of the staff in the endoscopy unit at King Abdulaziz University hospital, specifically regarding their help with all Fibroscan cases and for the data collection.

\section{References}

1. Chalasani N, Younossi Z, Lavine JE, Diehl AM, Brunt EM, Cusi K, et al. The diagnosis and management of non-alcoholic fatty liver disease: practice Guideline by the American Association for the Study of Liver Diseases, American College of Gastroenterology, and the American Gastroenterological Association. Hepatology. 2012;55(6):2005-23. doi: 10.1002/hep.25762. [PubMed: 22488764].
2. Soderberg C, Stal P, Askling J, Glaumann H, Lindberg G, Marmur J, et al. Decreased survival of subjects with elevated liver function tests during a 28-year follow-up. Hepatology. 2010;51(2):595-602. doi: 10.1002/hep.23314. [PubMed: 20014114].

3. Musso G, Gambino R, Cassader M, Pagano G. Meta-analysis: natural history of non-alcoholic fatty liver disease (NAFLD) and diagnostic accuracy of non-invasive tests for liver disease severity. Ann Med. 2011;43(8):617-49. doi: 10.3109/07853890.2010.518623. [PubMed: 21039302].

4. Vernon G, Baranova A, Younossi ZM. Systematic review: the epidemiology and natural history of non-alcoholic fatty liver disease and non-alcoholic steatohepatitis in adults. Aliment Pharmacol Ther. 2011;34(3):274-85. doi: 10.1111/j.1365-2036.2011.04724.x. [PubMed: 21623852].

5. Chen ZW, Chen LY, Dai HL, Chen JH, Fang LZ. Relationship between alanine aminotransferase levels and metabolic syndrome in nonalcoholic fatty liver disease. J Zhejiang Univ Sci B. 2008;9(8):616-22. doi: 10.1631/jzus.B0720016. [PubMed: 18763311].

6. Adams LA, Lymp JF, St Sauver J, Sanderson SO, Lindor KD, Feldstein A, et al. The natural history of nonalcoholic fatty liver disease: a population-based cohort study. Gastroenterology. 2005;129(1):113-21. [PubMed: 16012941].

7. Ong JP, Pitts A, Younossi ZM. Increased overall mortality and liver-related mortality in non-alcoholic fatty liver disease. $J$ Hepatol. 2008;49(4):608-12. doi: 10.1016/j.jhep.2008.06.018. [PubMed: 18682312].

8. Li H, Wang YJ, Tan K, Zeng L, Liu L, Liu FJ, et al. Prevalence and risk factors of fatty liver disease in Chengdu, Southwest China. Hepatobiliary Pancreat Dis Int. 2009;8(4):377-82. [PubMed:19666406].

9. Prashanth M, Ganesh HK, Vima MV, John M, Bandgar T, Joshi SR, et al. Prevalence of nonalcoholic fatty liver disease in patients with type 2 diabetes mellitus. J Assoc Physicians India. 2009;57:205-10. [PubMed: 19588648].

10. Leite NC, Salles GF, Araujo AL, Villela-Nogueira CA, Cardoso CR. Prevalence and associated factors of non-alcoholic fatty liver disease in patients with type-2 diabetes mellitus. Liver Int. 2009;29(1):113-9. doi: 10.1111/j.1478-3231.2008.01718.x. [PubMed: 18384521].

11. Akbar DH, Kawther AH. Nonalcoholic fatty liver disease in Saudi type 2 diabetic subjects attending a medical outpatient clinic: prevalence and general characteristics. Diabetes Care. 2003;26(12):3351-2. [PubMed: 14633828].

12. Al-hamoudi W, El-Sabbah M, Ali S, Altuwaijri M, Bedewi M, Adam M, et al. Epidemiological, clinical, and biochemical characteristics of Saudi patients with nonalcoholic fatty liver disease: a hospital-based study. Ann Saudi Med. 2012;32(3):288-92. doi: 10.5144/0256-4947.2012.288. [PubMed: 22588441].

13. Fallatah HI. Noninvasive biomarkers of liver fibrosis: an overview. Adv Hepatol. 2014;2014.

14. Machado MV, Cortez-Pinto H. Non-invasive diagnosis of non-alcoholic fatty liver disease. A critical appraisal. J Hepatol. 2013;58(5):1007-19. doi: 10.1016/j.jhep.2012.11.021. [PubMed: 23183525].

15. Lin ZH, Xin YN, Dong QJ, Wang Q, Jiang XJ, Zhan SH, et al. Performance of the aspartate aminotransferase-to-platelet ratio index for the staging of hepatitis C-related fibrosis: an updated meta-analysis. Hepatology. 2011;53(3):726-36. doi: 10.1002/hep.24105. [PubMed: 21319189].

16. Wai CT, Greenson JK, Fontana RJ, Kalbfleisch JD, Marrero JA, Conjeevaram $\mathrm{HS}$, et al. A simple noninvasive index can predict both significant fibrosis and cirrhosis in patients with chronic hepatitis C. Hepatology. 2003;38(2):518-26. doi: 10.1053/jhep.2003.50346. [PubMed: 12883497].

17. Sterling RK, Lissen E, Clumeck N, Sola R, Correa MC, Montaner J, et al. Development of a simple noninvasive index to predict significant fibrosis in patients with HIV/HCV coinfection. Hepatology. 2006;43(6):1317-25. doi:10.1002/hep.21178. [PubMed:16729309]. 
18. McPherson S, Stewart SF, Henderson E, Burt AD, Day CP. Simple non-invasive fibrosis scoring systems can reliably exclude advanced fibrosis in patients with non-alcoholic fatty liver disease. Gut. 2010;59(9):1265-9. doi: 10.1136/gut.2010.216077. [PubMed: 20801772].

19. Castera L, Vergniol J, Foucher J, Le Bail B, Chanteloup E, Haaser M, et al. Prospective comparison of transient elastography, Fibrotest, APRI, and liver biopsy for the assessment of fibrosis in chronic hepatitis $\mathrm{C}$. Gastroenterology. 2005;128(2):343-50. [PubMed: 15685546].

20. Wong VW, Vergniol J, Wong GL, Foucher J, Chan HL, Le Bail B, et al Diagnosis of fibrosis and cirrhosis using liver stiffness measurement in nonalcoholic fatty liver disease. Hepatology. 2010;51(2):454-62. doi: 10.1002/hep.23312. [PubMed: 20101745].

21. Lupsor M, Badea R, Stefanescu H, Grigorescu M, Serban A, Radu C, et al. Performance of unidimensional transient elastography in staging non-alcoholic steatohepatitis. J Gastrointestin Liver Dis. 2010;19(1):5360. [PubMed: 20361076].

22. Kumar R, Rastogi A, Sharma MK, Bhatia V, Tyagi P, Sharma P, et al. Liver stiffness measurements in patients with different stages of nonalcoholic fatty liver disease: diagnostic performance and clinicopathological correlation. Dig Dis Sci. 2013;58(1):265-74. doi: 10.1007/s10620012-2306-1. [PubMed: 22790906].

23. Friedrich-Rust M, Hadji-Hosseini H, Kriener S, Herrmann E, Sircar I, Kau A, et al. Transient elastography with a new probe for obese patients for non-invasive staging of non-alcoholic steatohepatitis. Eur Radiol. 2010;20(10):2390-6. doi:10.1007/s00330-010-1820-9. [PubMed: 20526777].

24. de Ledinghen V, Vergniol J, Foucher J, El-Hajbi F, Merrouche W, Rigalleau V. Feasibility of liver transient elastography with FibroScan using a new probe for obese patients. Liver Int. 2010;30(7):1043-8. doi: 10.1111/j.1478-3231.2010.02258.x. [PubMed: 20492500].

25. Akbar HO, Fallatah HI. Serum ALT levels in a cohort of healthy blood donors and volunteers from Saudi Arabia: the influence of sex and body mass index. Gastroentol Hepatol. 2010;1:13-9.

26. Poordad F. Review article: thrombocytopenia in chronic liver disease. Aliment Pharmacol Ther. 2007;26 Suppl 1:5-11. doi: 10.1111/j.13652036.2007.03510.x. [PubMed: 17958514].

27. Hancox SH, Smith BC. Liver disease as a cause of thrombocytopenia. QJM. 2013;106(5):425-31. doi: 10.1093/qjmed/hcs239. [PubMed: 23345462].

28. Yoneda M, Yoneda M, Fujita K, Inamori M, Tamano M, Hiriishi H, et al. Transient elastography in patients with non-alcoholic fatty liver disease (NAFLD). Gut. 2007;56(9):1330-1. doi: 10.1136/gut.2007.126417. [PubMed: 17470477].

29. Yoneda M, Yoneda M, Mawatari H, Fujita K, Endo H, Iida H, et al. Noninvasive assessment of liver fibrosis by measurement of stiffness in patients with nonalcoholic fatty liver disease (NAFLD). Dig Liver Dis 2008;40(5):371-8. doi: 10.1016/j.dld.2007.10.019. [PubMed:18083083].

30. Al-Nozha MM, Al-Maatouq MA, Al-Mazrou YY, Al-Harthi SS, Arafah MR, Khalil MZ, et al. Diabetes mellitus in Saudi Arabia. Saudi Med J. 2004;25(11):1603-10. [PubMed: 15573186].

31. Alqurashi KA, Aljabri KS, Bokhari SA. Prevalence of diabetes mellitus in a Saudi community. Ann Saudi Med. 2011;31(1):19-23. doi: 10.4103/0256-4947.75773. [PubMed: 21245594].

32. al-Nuaim AR, al-Rubeaan K, al-Mazrou Y, al-Attas O, al-Daghari N, Khoja T. High prevalence of overweight and obesity in Saudi Arabia. Int J Obes Relat Metab Disord. 1996;20(6):547-52. [PubMed: 8782731].

33. Al-Nozha MM, Al-Mazrou YY, Al-Maatouq MA, Arafah MR, Khalil MZ, Khan NB, et al. Obesity in Saudi Arabia. Saudi Med J. 2005;26(5):824-9. [PubMed: 15951877].

34. Razavizade M, Jamali R, Arj A, Talari H. Serum parameters predict the severity of ultrasonographic findings in non-alcoholic fatty liver disease. Hepatobiliary Pancreat Dis Int. 2012;11(5):513-20. [PubMed: 23060397].

35. Shimizu I. Chronic hepatitis C and B progress more slowly in females than males. In: Female hepatology: favorable role of female factors in liver disease. New York: Nova Biomedical Books; 2009. pp. 17-28.

36. Miyagi Y, Ishii Y, Nitta T, Ochi M, Shimizu K. Electrophysiological and histological assessment of transmurality after epicardial ablation using unipolar radiofrequency energy. J Card Surg. 2009;24(1):34-40. doi:10.1111/j.1540-8191.2008.00747.x. [PubMed:19040409].

37. Prati D, Taioli E, Zanella A, Della Torre E, Butelli S, Del Vecchio E, et al Updated definitions of healthy ranges for serum alanine aminotransferase levels. Ann Intern Med. 2002;137(1):1-10. [PubMed: 12093239].

38. Fallatah HI, Akbar HO. Lack of correlation between serum alanine amino transferrase and abdominal ultrasound in the diagnosis non alcoholic fatty liver disease in type 2 diabetes. Int J Diabetes Metab. 2010;18:120-3.

39. Dowman JK, Tomlinson JW, Newsome PN. Pathogenesis of nonalcoholic fatty liver disease. QJM. 2010;103(2):71-83.

40. Tilg H. Adipocytokines in nonalcoholic fatty liver disease: key players regulating steatosis, inflammation and fibrosis. Curr Pharm Des. 2010;16(17):1893-5. [PubMed: 20370678].

41. Jamali R, Arj A, Razavizade M, Aarabi MH. Prediction of Nonalcoholic Fatty Liver Disease Via a Novel Panel of Serum Adipokines. Medicine (Baltimore). 2016;95(5):ee2630. doi: 10.1097/MD.0000000000002630. [PubMed: 26844476].

42. Das SK, Balakrishnan V. Role of cytokines in the pathogenesis of nonalcoholic Fatty liver disease. Indian J Clin Biochem. 2011;26(2):202-9. doi: 10.1007/s12291-011-0121-7. [PubMed: 22468051]. 